\title{
A Study on a Pallarax Error and Its Dispersion on the Target
}

\author{
[Hwan Il Kang, Gun In Kim, Hyun Soo Kim]
}

\begin{abstract}
There are many errors to affect the system hit probability. Fixed bias, variable bias and random error are main categories for many errors. In this paper, a parallax error is considered. The parallax error belongs to the fixed bias. The fixed bias cannot be controlled by the shooting control device. The parallax error depends on the distance between the positions of the EOTS (electronic optical tracking device) and the gun. We calculate the error effect at the target by using the mil unit. The definition of a mil is based on a unit circle with a radius of one and 6400 mils mean the circumference in one turn. This analysis for the parallax error is a good guideline for design of the combat vehicle with gun having good system hit probability.
\end{abstract}

Keywords-system hit probability, combat vehicle, target, gun, fixed bias.

\section{Introduction}

To obtain system hit probability, we study error identification. The errors [1] are divided into three sets: fixed bias, variable bias and the random error. The fixed bias cannot be controlled by the shooting controlled device. The variable bias varies among different shot groups, while random error depends on the shooter and unexpected error. The parallax error belongs to the fixed bias. The fixed bias may be transferred to a mean value at the target. We want to calculate the error at the target due to the parallax error. The prediction of the system hit probability is obtained by using the normal distribution of the bullets on the target. The mean value of the normal distribution depends on the parallax error. The standard deviation values depend on the errors belongs to the variable bias and random error [2]. In Section II, we divide the errors into three categories. In Section III, we calculate the error at the target due to the parallax error. Conclusions will be followed in Section IV.

Hwan Il Kang and Hyun Soo Kim

Dept. of Information \& Communication Engineering, Myongji University Myongj-ro, Cheoin-gu, Yongin, Republic of Korea

Gun In Kim

Dept. of Acquisition, Konkuk University

120, Heung-dong-ro, Gwang-jin-gu, Seoul, Republic of Korea

\section{Three Kinds of Errors}

Each error[1] resulting in the hit probability of system can be divided into a three kinds of errors: fixed bias, variable bias and the random error. The fixed bias is the set of errors which cannot be controlled by the fire control device. A variable bias is the set of errors which are constant among single shot group and varies among different shot groups. The random error is a set of errors which result in random shots on the target. Fixed bias includes parallax error and drift error. Variable error includes aerodynamic jump error, cross wind error, range wind error, trunnion cant error and muzzle velocity error. The random error includes lay target error and round to round dispersion. In Table I, important errors are tabulated.

TABLE I. THREE TYPES OF ERRORS

\begin{tabular}{|c|c|c|}
\hline Fixed Bias & Variable Bias & $\begin{array}{c}\text { Random } \\
\text { Error }\end{array}$ \\
\hline \hline Parallax eror & $\begin{array}{c}\text { Aerodynamic } \\
\text { Jump error }\end{array}$ & $\begin{array}{c}\text { Lay Target } \\
\text { Center error }\end{array}$ \\
\hline Drift error & Cross Wind error & $\begin{array}{c}\text { Round to } \\
\text { Round } \\
\text { Dispersion }\end{array}$ \\
\hline & Range Wind error & \\
\hline & $\begin{array}{c}\text { Trunnion Cant } \\
\text { error }\end{array}$ & \\
\hline & $\begin{array}{c}\text { Muzzle Velocity } \\
\text { error }\end{array}$ & \\
\hline
\end{tabular}

\section{Main Results}

There is an error in the elevation angle due to the difference between the aiming device EOTS (Electronic Optical Tracking System) and the weapon equipment gun. The error in this case is referred to as the parallax error. If we can derive the parallax error in the figure 1 . The parallax error is

$$
E_{P A X}=g-f
$$

In the figure 1, the equation is obtained

$$
r(p \tan (f)-h)=p(r \tan (g)-h)
$$


Rewriting the above equation

$$
g=\tan ^{-1}\left(\left(\tan (f)-\frac{h}{p}\right)+\frac{h}{r}\right)
$$

The parallax error is $g-e$, but compensating by $f-$ $e$, the resulting parallax is $(g-e)-(f-e)=g-f$. The angle $f$ is

$$
\operatorname{ptan}(e)=\operatorname{ptan}(f)-h
$$

Rewriting the equation (4),

$$
f=\tan ^{-1}\left(\tan (e)+\frac{h}{p}\right)
$$

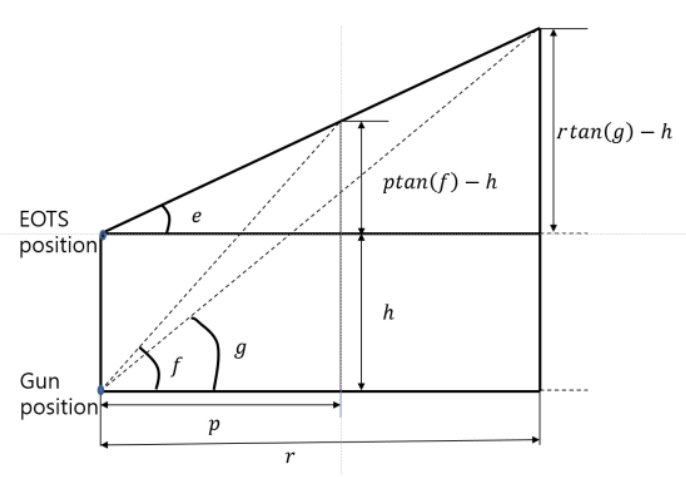

Figure 1. Parallax error with Gun and EOTS

Thus

$$
g-f=\tan ^{-1}\left(\left(\tan (f)-\frac{h}{p}\right)+\frac{h}{r}\right)-\tan ^{-1}\left(\tan (e)+\frac{h}{p}\right)
$$

Using the equation

$$
r \tan (f)=(h+p \tan (e)) / p
$$

we obtain

$$
g-f=\tan ^{-1}\left(\left(\tan (e)+\frac{h}{r}\right)-\tan ^{-1}\left(\tan (e)+\frac{h}{p}\right)\right)
$$

Thus the parallax error equation with mil unit is

$$
E_{p}=\frac{6400}{2 \pi} \tan ^{-1}\left(\left(\tan (e)+\frac{h}{r}\right)-\tan ^{-1}\left(\tan (e)+\frac{h}{p}\right)\right)
$$

We assume that the parallax error is zero at range of $1200 \mathrm{~m}$. Table II tabulates the parallax error at the target for different ranges and elevation angles.

TABLE II. PARALLAX ERROR VAUES

\begin{tabular}{|c|c|c|c|}
\hline $\begin{array}{c}\text { Range } \\
\text { Distance } \\
\text { (m) }\end{array}$ & $\mathbf{1 0 0 0}$ & $\mathbf{1 5 0 0}$ & $\mathbf{2 0 0 0}$ \\
\hline \hline $\begin{array}{c}\text { Elevation } \\
\text { angle }\end{array}$ & \multicolumn{3}{|c|}{5} \\
\hline$E_{p}[\mathrm{mil}]$ & 0.1804 & -0.3207 & -0.4059 \\
\hline \hline $\begin{array}{c}\text { Elevation } \\
\text { angle }\end{array}$ & 10 \\
\hline$E_{p}[\mathrm{mil}]$ & 0.1762 & -0.3133 & -0.3966 \\
\hline \hline
\end{tabular}

\section{Conclusions}

In the case of a fixed bias parallax error, a high angle error occurs when the position of the EOTS and the gun are apart. If the positions of the EOTS and the gun are apart in the vertical direction, the error according to each elevation angle is obtained. For example, if the range is 2000 and the elevation angle is 10 degrees, an error of about -0.3966 mil appears. This analysis for the parallax error is a good guideline for design of the combat vehicle with gun having good system hit probability.

\section{Acknowledgment}

This work was supported by the Research program (the specialized research center for future ground system analysis (Contract No. 311165-911074201) funded by the agency for defense development in the republic of Korea.

\section{References}

[1] G. I. Kim, H. I. Kang and J. J. Pyun, "Delivery accuracy determination for indirect weapon systems using error budget methodology," International Conference on Convergency Technology, July, Hokkaido, Japan, 2017. 
[2] H. I. Kang, G. I. Kim, and H. S. Kim, "A study on prediction of probability of hit for an anti-aircraft artillery," Indian Journal of Science and Technology, vol. 9, no. 1, Dec., 2016.

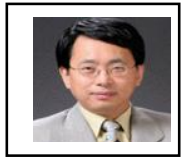

HWAN IL KANG was born in Incheon, South Korea and graduated from the Seoul National University, Korea Advanced Institute of Science \& Technology and University of Wisconsin-Madison in USA where he studied electrical and computer engineering and obtained his BS, MS and $\mathrm{Ph}$. D. degrees, respectively. He has been a professor at dept. of Information \& Communications eng. in Myongji University, South Korea since 1996. His research interest includes modelling \& simulation, military sciences and control applications. 